\title{
ChemComm
}

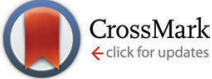

Cite this: Chem. Commun., 2015 51,16526

Received 2nd July 2015, Accepted 22nd September 2015

DOI: $10.1039 / \mathrm{c5cc05450h}$

www.rsc.org/chemcomm

\section{Nucleic-acid recognition interfaces: how the greater ability of RNA duplexes to bend towards the surface influences electrochemical sensor performance $\dagger$}

\author{
Roya Tavallaie, ${ }^{\text {abc Nadim Darwish, }}{ }^{a}$ D. Brynn Hibbert ${ }^{\mathrm{a}}$ and J. Justin Gooding *abc
}

The influence of RNA versus DNA on the performance of electrochemical biosensors where redox-labelled nucleic acid duplexes bend towards the electrode surface has been assessed. Faster electron transfer was observed for duplexes containing RNA, suggesting duplexes with RNA are more flexible. These data are of particular importance for microRNA biosensors.

Surface-bound nucleic acids have application in molecular devices and therapeutics. ${ }^{1-4}$ The performance of such interfaces is to a large extent affected by biophysical properties of nucleicacids such as the persistence length and conformation. ${ }^{5,6}$ Understanding of, and control over, nucleic acids-surface interactions and hybridization events have been of a great importance for improving devices that employ surface-bound nucleic-acids. ${ }^{7-11}$ In the last few years there has been an increase in the application of surface bound nucleic acids for the detection of RNA sequences using the exact same experimental constructs as used for DNA sequences. RNA combines the information storage capabilities of DNA with additional regulatory capabilities. ${ }^{12}$ This increase in interest in detecting RNA sequences arises from the discovery of microRNA, ${ }^{13,14}$ an important class of non-coding RNAs that play a role in the regulation and dysregulation of many diseases. The question therefore arises as to whether sensing concepts for the detection of DNA are directly translatable to the detection of RNA and/or whether the analytical performance of the final sensor is influenced by the target being RNA and not DNA.

The questions above arise because although RNA shares many common structural features with DNA, the presence of a

\footnotetext{
${ }^{a}$ School of Chemistry, The University of New South Wales, Sydney, NSW 2052, Australia. E-mail: Justin.gooding@unsw.edu.au; Fax: +61 29385 6141; Tel: +61293855384

${ }^{b}$ Australian Centre for NanoMedicine, The University of New South Wales, Sydney, NSW 2052, Australia

${ }^{c} A R C$ Centre of Excellence in Convergent Bio-Nano Science and Technology,

The University of New South Wales, Sydney, NSW 2052, Australia

$\dagger$ Electronic supplementary information (ESI) available: Experimental methods, cyclic voltammograms, square wave voltammograms and impedance data. See DOI: $10.1039 / \mathrm{c} 5 \mathrm{cc} 05450 \mathrm{~h}$
}

hydroxyl group at the $2^{\prime}$ position of the ribose sugar causes the RNA double helix to adopt the A-form conformation rather than the B-form conformation, which is most commonly observed in DNA double helix. RNA duplexes have been found to be different from their equivalent DNA duplexes in properties such as thermodynamic stability, hydration and flexibility. ${ }^{15,16}$ Conceptually, flexibility denotes the ability of a given structure to be deformed. This concept needs to be applied with caution for nucleic acids. This is because of the word flexibility being applied in two ways in the nucleic acid literature. One is local dynamics in the backbone and the second is global deformations in double helix. ${ }^{16}$ Global deformations in DNA and RNA duplexes, including tilt, roll, twist and bend have been extensively studied in solution, but there are few studies of the impact of global deformations of nucleic acids duplexes on device performance for surface bound nucleic acids. ${ }^{9,17,18}$ So far all the techniques utilized for RNA detection assume the properties of surface-bound RNA duplexes, such as their ability to bend towards the surface, the same as their equivalent DNA ones.

This study addresses the question of the impact of RNA targets, as distinct from DNA, using one of the classical electrochemical DNA biosensing constructs as depicted in Fig. 1. In this construct the surface bound nucleic acid probe possesses a methylene blue (MB) redox label at its distal end which can transfer electrons to the underlying electrode when the MB is in close proximity to the electrode. ${ }^{18}$ Upon hybridization with a target nucleic acid sequence the increased persistence length of the duplex decreases the apparent rate of electron transfer between the $\mathrm{MB}$ and the electrode because the residence time of the MB near the electrode is reduced. ${ }^{19}$ This transduction mechanism makes this experimental construct ideal for exploring the impact of RNA on device performance as the apparent rate of electron transfer is sensitive to the global deformability of the surfacebound redox-labelled nucleic acids. ${ }^{5,20-22}$ Interfaces have been designed using either 22-base pairs probe DNA or the equivalent sequence composed of RNA. The target was either the microRNA sequence, miR-21, or the corresponding DNA sequences. miR-21 is one of the most frequently studied cancer-related microRNAs 

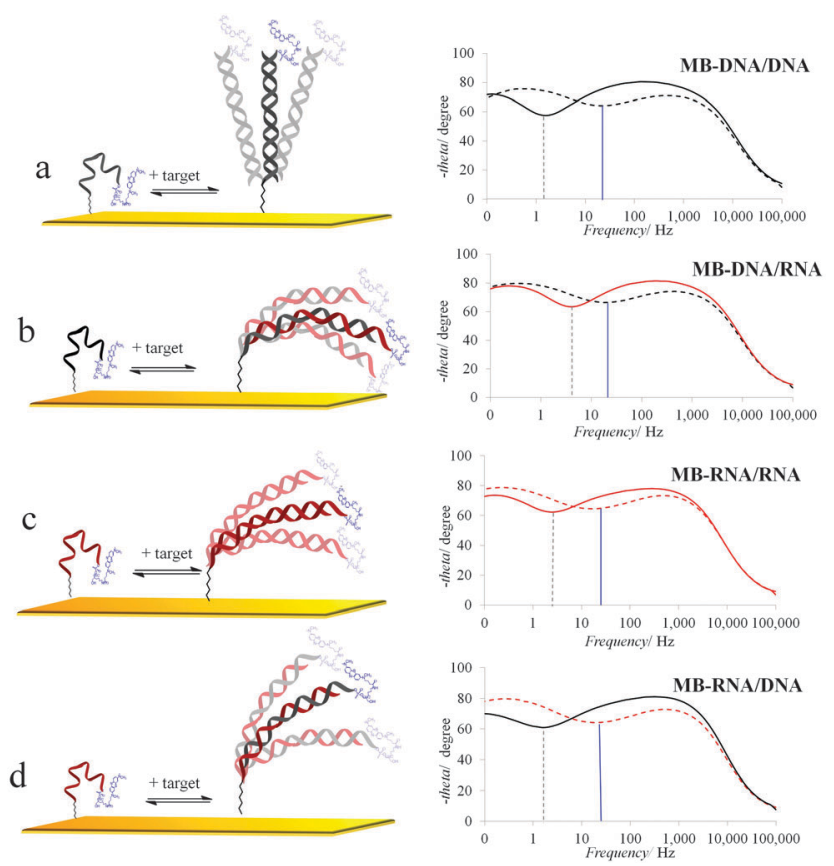

Fig. 1 Schematic representing probe DNA (black)/RNA (red) modified electrodes before and after hybridization with target DNA/RNA resulting in four systems a: MB-DNA/DNA, b: MB DNA/RNA, c: MB-RNA/RNA, d: MB-RNA/ DNA. Formation of more rigid duplex after hybridization results in an increase in the average distance of MB from the surface. Corresponding Bode plots obtained for each system before (dashed lines) and after (solid lines) hybridization are presented at the right side.

which is dysregulated in most cancers by acting as a key regulator of oncogenic processes. ${ }^{23-25}$ Hence four different interfaces were investigated, MB-DNA/DNA, MB-DNA/RNA, MB-RNA/ RNA and MB-RNA/DNA (the schematic presented at Fig. 1).

The DNA/DNA system was used here as a reference system, having been extensively studied, to compare the properties of the studied systems. Otherwise identical surface chemistry was employed where gold electrodes are modified with thiol bearing nucleic-acids followed by separating the DNA strands using 6-mercaptohexanol; the so called Tarlov interface. ${ }^{4}$ The detailed procedures for fabricating the sensing interfaces are described in the ESI. $\dagger$ The average surface probe density was calculated by integrating the cyclic voltammetry peak corresponding to its redox reaction. The average coverage for probe DNA/RNA was estimated, from the charge passed during cyclic voltammetry, to be $(1.29 \pm 0.21) \times 10^{12}$ molecules $\mathrm{cm}^{-2}$.

As could be inferred from the cyclic voltammograms presented in Fig. S1 (ESI $\dagger$ ), all four systems displayed obvious changes in the peak current and peak-to-peak separation upon hybridization with target. Such changes are indicative of slower electron transfer kinetics for surface-bound duplexes relative to surface-bound single strand nucleic-acids. Both at relatively low $\left(1 \mathrm{~V} \mathrm{~s}^{-1}\right)$ and high $\left(4 \mathrm{~V} \mathrm{~s}^{-1}\right)$ scan rates, the hybridization-induced peak-to-peak separation decreased in the order of MB-DNA/DNA > MB-RNA/DNA > MB-RNA/RNA > MB-DNA/RNA. This order reflects the relative ease by which the $\mathrm{MB}$ can access the electrode surface. In the case of both the DNA/DNA and DNA/RNA, which are the two extremes in the above order, the hybridization efficiency was estimated using a chronocoulometry method ${ }^{26}$ to be $88 \pm 8 \%$ and $82 \pm 11 \%$. Note the MB-RNA/DNA and MB-RNA/RNA show similar peak separations to the MB-DNA/RNA suggesting similar degrees of hybridization.

To establish the kinetics of electron transfer, and the change associated with duplex formation, electrochemical impedance spectroscopy (EIS) ${ }^{27-29}$ was performed at an applied DC potential of $275 \mathrm{mV}$ that matches the apparent formal of MB. The EIS results can be presented as Bode-plots (Fig. 1) which allow for a rapid visualization of the kinetics data. ${ }^{30,31}$ At an AC frequency that approaches the time constant of the redox reaction, the value of the phase angle decreases and reaches a minimum. The frequency at which the phase angle minimum occurs can be used to qualitatively differentiate between systems with different electron-transfer kinetics. Systems with the phase angle minimum occurring at higher frequencies have faster rates of electron transfer. ${ }^{27,30,31}$ As can be seen in Fig. 1, the Bode plots obtained for either probe DNA or probe RNA modified electrodes (before hybridization with a target) exhibit a very similar phase angle minimum.

Upon hybridization to form a duplex, in all cases a shift in the phase angle minimum to the lower frequencies occurs as expected with the duplexes being more rigid than single strands such that the redox species cannot access the electrode surface as easily. ${ }^{27}$ The kinetics parameters were quantified by fitting the EIS to an equivalent of the Randles circuit model ${ }^{29}$ using the complex non-linear least-squares technique (Fig. S2 and Table S1, ESI $\dagger$ ). As shown in Table 1, each system exhibited a lower value for the apparent rate constant of electron transfer after hybridization with target, compared to before hybridization.

Table 1 Summary of the average change in the SWV peak current and kinetics of electron transfer upon introducing mismatch base pairs

\begin{tabular}{|c|c|c|}
\hline$k_{\text {unhybridized }} / k_{\text {hybridized }}$ & & $\begin{array}{l}\left(\text { SWV suppression }{ }^{2}\right) / \\
(\text { SWV suppression })_{\text {target }}\end{array}$ \\
\hline \multicolumn{3}{|l|}{ MB-DNA/DNA } \\
\hline Complementary & $11.53 \pm 0.11$ & $\mathbf{\sigma}_{1.00}$ \\
\hline Single MM & $7.90 \pm 0.09$ & 0.93 \\
\hline Triple MM & $1.24 \pm 0.03$ & 0.15 \\
\hline
\end{tabular}

\section{MB-DNA/RNA}

Complementary

Single MM

Triple MM

$4.62 \pm 0.06$
$1.75 \pm 0.03$
$1.48 \pm 0.02$

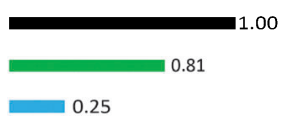

MB-RNA/DNA

Complementary

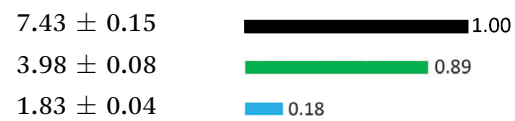

Triple MM

$1.83 \pm 0.04$

0.18

\section{MB-RNA/RNA}

Complementary

Single MM

Triple MM

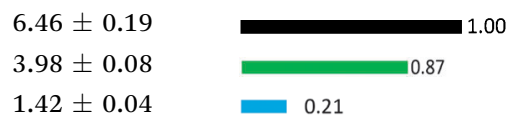

Relative change in the SWV current $\%=\left(I_{\text {unhybridized }}-I_{\text {hybridized }}\right) / I_{\text {unhybridized }}$. 
The ratio $k_{\text {unhybridized }} / k_{\text {hybridized }}$ was $11.53 \pm 0.11$ for the MB-DNA/ DNA, $4.62 \pm 0.06$ for MB-DNA/RNA, $6.46 \pm 0.19$ for MB-RNA/RNA, and $7.43 \pm 0.15$ for MB-RNA/DNA. In agreement with the order of change in the rate constant values $\left(k_{\text {unhybridized }} / k_{\text {hybridized }}\right)$, a greater change in the square wave voltammetry current was observed for the DNA/DNA duplex compared with three other systems containing RNA (Fig. S3 and S4, ESI $\dagger$ ). The lesser values of $k_{\text {unhybridized }} / k_{\text {hybridized }}$ obtained for systems containing RNA, as probe, target or both, suggests that presence of RNA in the duplex enhances the ability of the redox label MB to access the electrode surface after hybridization.

This result is in good agreement with studies related to the global deformability of nucleic acid duplexes in solution. ${ }^{15}$ RNA/RNA is known to form a A-form duplex conformation with more global deformations compared to the B-form DNA/DNA duplex conformation. ${ }^{32}$ Hybrids of DNA/RNA and RNA/DNA are known to form conformations between B-form and A-form conformations, but with more the character of the A-form conformation. ${ }^{33,34}$

Although MB-DNA/RNA and MB-RNA/DNA systems behaved more similarly to the MB-RNA/RNA system, than the MB-DNA/ DNA system, they do show differences in electrochemical properties. Having the same composition, different $k_{\text {unhybridized }} / k_{\text {hybridized }}$ values for MB-DNA/RNA and MB-RNA/DNA systems demonstrate the impact of the choice of probe and target being DNA or RNA on the properties of surface-bound nucleic acids. From an analytical perspective the result indicates for the detection of miRNAs, a DNA probe strand on the surface gives a higher discrimination between single strands and duplexes than a RNA probe strand.

Next, the effect of introducing mismatched base pairs was investigated (Fig. S5, ESI $\dagger$ ). Each system showed a decrease in $k_{\text {unhybridized }} / k_{\text {hybridized }}$ values upon introducing mismatch sequence relative to a complementary sequence. This observation, which is in agreement with the results of a study on DNA mismatched duplexes, ${ }^{17}$ suggests that the duplex can more easily access to the surface upon introducing a single mismatch base pair. A single mismatch in the middle base pair of the duplex has been shown to divide the duplex into two segments, which behave like rigid rods being connected by the mismatch as a flexible hinge. ${ }^{35}$ The $k_{\text {unhybridized }} / k_{\text {hybridized }}$ values in duplexes containing RNA are affected more by the introduction of a single mismatch base pair than in the MB-DNA/DNA system (Table 1). As an example, the $k_{\text {unhybridized }} / k_{\text {hybridized }}$ value for MB-DNA/DNA decreased from $11.53 \pm 0.11$ to $7.90 \pm 0.09$ upon introducing a single mismatch base pair while for MB-DNA/RNA the decrease was $4.62 \pm 0.06$ to $1.75 \pm 0.03$; a much larger percentage change for the MB-DNA/ RNA system $(-62 \%)$ than the $-31 \%$ for MB/DNA-DNA. These results show that presence of a mismatch base pair has a higher impact on the ability of duplexes containing RNA to bend towards the surface, compared to their equivalent DNA/DNA duplex. Again this analytically important as it shows that it will be easier to determine mismatches using RNA targets than DNA targets.

To summarize, two recognition interfaces composed of MBlabelled probe DNA or RNA and mercaptohexanol were exposed to the target DNA and RNA (miR-21) to give the four systems: MB-DNA/DNA, MB-DNA/RNA, MB-RNA/RNA and MB-RNA/DNA.
Introducing RNA in the structure of the duplex (as probe, target or both) was found to result in a decrease in the average distance of the redox label from the surface after hybridization, enabling faster electron transfer kinetics, compared to the reference DNA/DNA system. These data suggest a greater ability of the surface-bound duplexes containing RNA to approach towards the surface, compared to DNA/DNA duplex. Comparing the hybridization-induced change in the electrochemical properties of mismatched duplexes with those made using a complementary target also revealed that introducing a single base pair mismatch has a more pronounced effect on the average distance of redox label from the surface for duplexes containing RNA, compared to the MB-DNA/DNA system.

The importance of these results relate to the recent trend of exploring different approaches for the detection of microRNA, as a blood-based cancer biomarker. Most approaches are simply variants of biosensors for detecting DNA. Therefore, our observation that the choice of probe and target being RNA or DNA has an impact on the ability of both matched and mismatched duplexes to bend towards the surface is of particular relevance to the rational design of microRNA biosensors, where having a good understanding and control on the properties of surface-bound RNA duplexes is necessary for achieving good sensitivity and selectivity. These results show that for detecting microRNA the best interfacial configuration is using a DNA probe strand to detect the target miRNA strand as this will give a better selectivity than having a probe RNA sequence. However this configuration has the lowest discrimination between single strands and double strands.

We thank the University of New South Wales and Australian Research Council Centre of Excellence in Convergent Bio-Nano Science and Technology (CE140100036) for funding.

\section{Notes and references}

1 Y. Krishnan and F. C. Simmel, Angew. Chem., Int. Ed., 2011, 50, 3124-3156.

2 A. E. Prigodich, A. H. Alhasan and C. A. Mirkin, J. Am. Chem. Soc., 2011, 133, 2120-2123.

3 H. Yang, A. Hui, G. Pampalakis, L. Soleymani, F. F. Liu, E. H. Sargent and S. O. Kelley, Angew. Chem., Int. Ed., 2009, 48, 8461-8464.

4 J. Wu, S. Balasubramanian, D. Kagan, K. M. Manesh, S. Campuzano and J. Wang, Nat. Commun., 2010, 1.

5 F. J. Mearns, E. L. S. Wong, K. Short, D. B. Hibbert and J. J. Gooding, Electroanalysis, 2006, 18, 1971-1981.

6 E. M. Boon and J. K. Barton, Bioconjugate Chem., 2003, 14, 1140-1147.

7 E. L. S. Wong, E. Chow and J. J. Gooding, Langmuir, 2005, 21, 6957-6965.

8 K. J. Odenthal and J. J. Gooding, Analyst, 2007, 132, 603-610.

9 E. Farjami, R. Campos and E. E. Ferapontova, Langmuir, 2012, 28, 16218-16226.

10 A. W. Peterson, L. K. Wolf and R. M. Georgiadis, J. Am. Chem. Soc., 2002, 124, 14601-14607.

11 R. Tavallaie, N. Darwish, M. Gebala, D. B. Hibbert and J. J. Gooding, ChemElectroChem, 2014, 1, 165-171.

12 W. Gilbert, Nature, 1986, 319, 618.

13 H. F. Dong, J. P. Lei, L. Ding, Y. Q. Wen, H. X. Ju and X. J. Zhang, Chem. Rev., 2013, 113, 6207-6233.

14 R. Tavallaie, S. R. M. De Almeida and J. J. Gooding, Wiley Interdiscip. Rev.: Nanomed. Nanobiotechnol., 2014, 7, 580-592.

15 S. Pramanik, S. Nagatoishi, S. Saxena, J. Bhattacharyya and N. Sugimoto, J. Phys. Chem. B, 2011, 115, 13862-13872.

16 A. Perez, A. Noy, F. Lankas, F. J. Luque and M. Orozco, Nucleic Acids Res., 2004, 32, 6144-6151. 
17 R. Ikeda, S. Kobayashi, J. Chiba and M. Inouye, Chem. - Eur. J., 2009, $15,4822-4828$.

18 N. Husken, M. Gebala, F. La Mantia, W. Schuhmann and N. MetzlerNolte, Chem. - Eur. J., 2011, 17, 9678-9690.

19 R. J. White and K. W. Plaxco, Anal. Chem., 2010, 82, 73-76.

20 A. A. Lubin, R. Y. Lai, B. R. Baker, A. J. Heeger and K. W. Plaxco, Anal. Chem., 2006, 78, 5671-5677.

21 A. Anne and C. Demaille, J. Am. Chem. Soc., 2006, 128, 542-557.

22 A. Anne and C. Demaille, J. Am. Chem. Soc., 2008, 130, 9812-9823.

23 X. Zhou, X. P. Wang, Z. B. Huang, J. Wang, W. Zhu, Y. Q. Shu and P. Liu, PLoS One, 2014, 9, e102413.

24 P. P. Medina, M. Nolde and F. J. Slack, Nature, 2010, 467, U86-U119. 25 S. D. Selcuklu, M. T. A. Donoghue and C. Spillane, Biochem. Soc. Trans., 2009, 37, 918-925.

26 A. B. Steel, T. M. Herne and M. J. Tarlov, Bioconjugate Chem., 1999, 10, 419-423.
27 E. Laviron, J. Electroanal. Chem., 1979, 105, 35-42.

28 S. E. Creager and T. T. Wooster, Anal. Chem., 1998, 70, 4257-4263.

29 J. R. Macdonald and L. D. Potter, Solid State Ionics, 1987, 24, 61-79.

30 A. D. Abhayawardhana and T. C. Sutherland, J. Phys. Chem. C, 2009, 113, 4915-4924.

31 S. Ciampi, M. James, M. H. Choudhury, N. A. Darwish and J. J. Gooding, Phys. Chem. Chem. Phys., 2013, 15, 9879-9890.

32 A. Noy, A. Perez, F. Lankas, F. Javier Luque and M. Orozco, J. Mol. Biol., 2004, 343, 627-638.

33 J. I. Gyi, A. N. Lane, G. L. Conn and T. Brown, Biochemistry, 1998, 37, 73-80.

34 Z. Shakked, G. Guersteinguzikevich, M. Eisenstein, F. Frolow and D. Rabinovich, Nature, 1989, 342, 456-460.

35 N. Husken, M. Gebala, A. Battistel, F. La Mantia, W. Schuhmann and N. Metzler-Nolte, ChemPhysChem, 2012, 13, 131-139. 